\title{
Enhancement of $T_{c}$ by disorder in underdoped iron pnictide superconductors
}

\author{
R. M. Fernandes ${ }^{1,2}$ M. G. Vavilov, ${ }^{3}$ and A. V. Chubukov ${ }^{3}$ \\ ${ }^{1}$ Department of Physics, Columbia University, New York, New York 10027, USA \\ ${ }^{2}$ Theoretical Division, Los Alamos National Laboratory, Los Alamos, NM, 87545, USA \\ ${ }^{3}$ Department of Physics, University of Wisconsin, Madison, Wisconsin 53706, USA
}

(Dated: January 21, 2020)

\begin{abstract}
We analyze how disorder affects the transition temperature $T_{c}$ of the $s^{+-}$superconducting state in the iron pnictides. The conventional wisdom is that $T_{c}$ should rapidly decrease with increasing interband non-magnetic impurity scattering, but we show that this behavior holds only in the overdoped region of the phase diagram. In the underdoped regime, where superconductivity emerges from a pre-existing magnetic state, disorder gives rise to two competing effects: breaking of the Cooper pairs, which tends to reduce $T_{c}$, and suppression of the itinerant magnetic order, which tends to bring $T_{c}$ up. We show that for a wide range of parameters the second effect wins, i.e. in the coexistence state $T_{c}$ can increase with disorder. Our results provide an explanation for several recent experimental findings and lend additional support to $s^{+-}$-pairing in the iron pnictides.
\end{abstract}

Introduction. The symmetry of the superconducting state of the iron-based superconductors (FeSCs) is still a subject of intense debate ${ }^{1}$. Photoemission experiments on moderately-doped FeSCs show quite convincingly 2 that the pairing state is $s$-wave, i.e. fully gapped. However, since the FeSCs are multi-band systems, the $s$-wave superconducting state (SC) can have either $s^{++}$ symmetry, if the gaps on different Fermi-surface pockets have the same sign, or $s^{+-}$symmetry, if the gaps on different pockets have opposite signs ${ }^{3}$. The $s^{+-}$state emerges due to a repulsive inter-band interaction enhanced by spin-fluctuations - this is the key element in the theories of a magnetic pairing mechanism in the FeSCs $\stackrel{4}{*}$. On the other hand, the $s^{++}$state emerges if the inter-band interaction is attractive and is enhanced by orbital fluctuations $\underline{\underline{5}}$

A seemingly straightforward way to distinguish between $s^{+-}$and $s^{++}$pairing symmetries is their responses to impurity scattering. Both SC states are nearly unaffected by intra-band scattering. However, while interband scattering is harmless to the $s^{++}$state, it is pairbreaking to the $s^{+-}$state, leading to a suppression of $T_{c} \stackrel{\underline{6}}{\underline{\underline{6}}}$ To verify this experimentally, one has to choose a dopant that acts predominantly as a non-magnetic impurity scatterer. In the FeSCs, this is not a trivial task, as many transition-metal dopants significantly change the carrier concentration $\underline{\underline{7}}$

One direction explored by many groups was to substitute $\mathrm{Zn}$ for Fe. Early data on LaFeAs $\left(\mathrm{O}_{1-x} \mathrm{~F}_{x}\right)$ showed that $T_{c}$ weakly depends on the $\mathrm{Zn}$ concentration ${ }^{8}$, and were interpreted as an evidence in favor of an $s^{++}$state. Subsequent studies $\stackrel{9,10}{ }$, however, found that the effect of Zn substitution depends on the doping level $x$ : while in the overdoped regime $T_{c}$ displays a sharp decrease, in agreement with what is expected for an $s^{+-}$state, at optimal doping $T_{c}$ remains virtually the same. More surprisingly, in the underdoped regime of $\mathrm{LaFeAs}\left(\mathrm{O}_{1-x} \mathrm{~F}_{x}\right)$, $T_{c}$ increases with $\mathrm{Zn}$ concentration ${ }^{9}$, an observation that is puzzling not only for an $s^{+-} \mathrm{SC}$ state, but even for a conventional $s^{++}$state. A similar increase of $T_{c}$ with disorder was found in the underdoped mate- rial $\mathrm{Ba}\left(\mathrm{Fe}_{1-x} \mathrm{Co}_{x}\right)_{2} \mathrm{As}_{2}$ with substitution of $\mathrm{Cu}$ for $\mathrm{Fe} \underline{11}$. That the dopant $\mathrm{Cu}$ atoms act as impurity-scatterers follows from both band structure calculations $\underline{\underline{6}}$ and neutron scattering experiments $\frac{12}{2}$ Intriguingly, measurements in the same $\mathrm{Ba}\left(\mathrm{Fe}_{1-x} \mathrm{Co}_{x}\right)_{2} \mathrm{As}_{2}$ materials, but with $\mathrm{Zn}$ replacing $\mathrm{Fe}$, showed that $T_{c}$ decreases with increasing $\mathrm{Zn}$ concentration even in the underdoped region ${ }^{13}$, although $T_{c}$ decreases faster in the overdoped region.

In this paper, we describe the effect of disorder on the superconducting transition temperature $T_{c}$ of the $s^{+-}$ state in underdoped samples, when superconductivity develops in the presence of SDW order. We argue that the conventional wisdom that $T_{c}$ decreases with increasing impurity concentration does not work in the underdoped region. Indeed, even in a clean system, the reason why $T_{c}$ goes down deep in the underdoped region is because SDW order competes with superconductivity. As doping increases, the SDW order becomes weaker, and $T_{c}$ increases $\stackrel{14,15}{=}$ When disorder is added at a fixed doping concentration, it influences SDW and SC orders differently: while both intra-band and inter-band impurity scattering weaken SDW $\stackrel{16}{\longleftarrow}$ only inter-band scattering is pair-breaking for $s^{+-}$superconductivity $\underline{6,17}$ As a result, disorder without inter-band scattering component does not directly affect SC pairing, but weakens the SDW order, leading to an increase in $T_{c}$. The situation is more complicated when both intra-band and inter-band scattering components are present. In this situation, impurity scattering affects $T_{c}$ both directly, via pair-breaking, and indirectly, via the suppression of SDW. Therefore, the two effects push $T_{c}$ in opposite directions, and whether $T_{c}$ increases or decreases with increasing impurity concentration depends on the interplay between the system parameters.

Our key results are summarized in Fig. 1, where we compare the phase diagrams of FeSCs with and without impurities for two representative values of the SDW and SC couplings and for on-site impurity potential (i.e. equal intra-band and inter-band impurity scatterings). For one set of parameters, Fig. 1(a), $T_{c}$ increases with increasing disorder in the underdoped region. This be- 

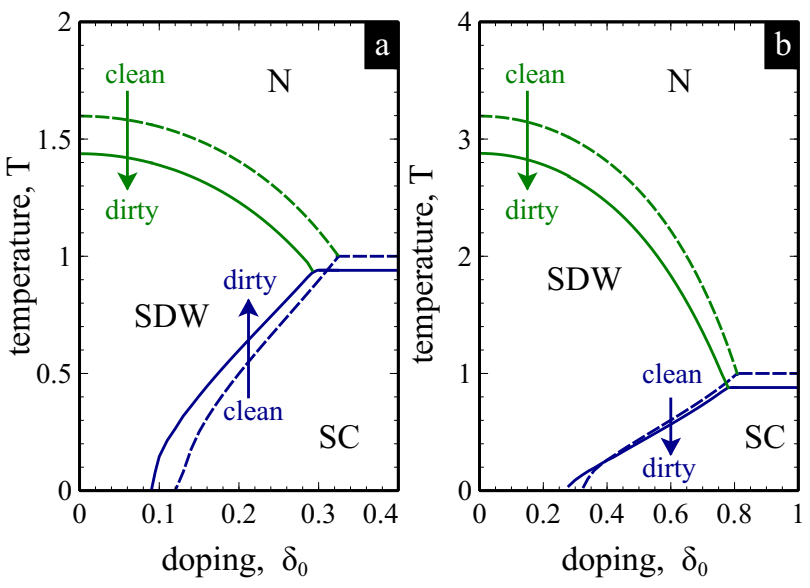

Figure 1: (Color online) Temperatures of normal (N)-toSDW, SDW-to-SC and N-to-SC transitions as functions of doping for the clean and dirty cases (dashed and solid lines, respectively) for two sets of system parameters. In the underdoped region, where SC emerges from a pre-existing SDW phase, $T_{c}$ for $s^{+-}$pairing increases with the concentration of non-magnetic impurities for one set of parameters (panel a) and weakly decreases for the other set (panel b). These two behaviors are consistent with the data in Refs. 9 11 and Ref. [13], respectively. Temperatures and $\delta_{0}$ are measured in units of $T_{c, 0}$, which is the SC transition temperature at perfect nesting and without SDW (for pure SDW, the corresponding temperature is $\left.T_{N, 0}\right)$. We used in (a): $T_{N, 0} / T_{c, 0}=2, \delta_{2} /\left(2 \pi T_{c, 0}\right)=0.4$, and impurity-scattering amplitudes $\Gamma_{0}=\Gamma_{\pi}=0.006\left(2 \pi T_{c, 0}\right) ;$ and in (b): $T_{N, 0} / T_{c, 0}=$ $4, \delta_{2} /\left(2 \pi T_{c, 0}\right)=0.8$, and $\Gamma_{0}=\Gamma_{\pi}=0.012\left(2 \pi T_{c, 0}\right)$.

havior provides an explanation for the experimental results of Refs. [9,11]. For the other set of parameters, Fig. 1(b), $T_{c}$ decreases with disorder in the underdoped region, but with a smaller rate than in the overdoped region. This behavior is consistent with the experiments in Ref. 13].

We also analyze the dependence of $T_{c}$ on the ratio of intra-band and inter-band scattering rates (see Fig. 2). For some parameters, as those in Fig. 1(a), $T_{c}$ increases for any ratio of intra-band and inter-band impurityscattering. For other parameters, as those in Fig. 1(b), $T_{c}$ increases when intra-band scattering dominates and decreases when inter-band scattering dominates. Even in the latter case, the rate at which $T_{c}$ decreases with the strength of disorder is smaller than in a pure $s^{+-}$ superconductor.

The model We follow earlier works $14,15,19$ and consider a minimal two-band model for the interplay between itinerant SDW and $s^{+-}$SC. In particular, we consider a circular hole pocket at the center of the Fe-only Brillouin zone, and an elliptical electron pocket displaced from the center by $\mathbf{Q}=(\pi, 0)$ (or $(0, \pi))$. The non-interacting fermionic Hamiltonian is

$$
\mathcal{H}_{0}=\sum_{\mathbf{k} \sigma} \varepsilon_{1, \mathbf{k}} c_{\mathbf{k} \sigma}^{\dagger} c_{\mathbf{k} \sigma}+\sum_{\mathbf{k} \sigma} \varepsilon_{2, \mathbf{k}} f_{\mathbf{k} \sigma}^{\dagger} f_{\mathbf{k} \sigma}
$$

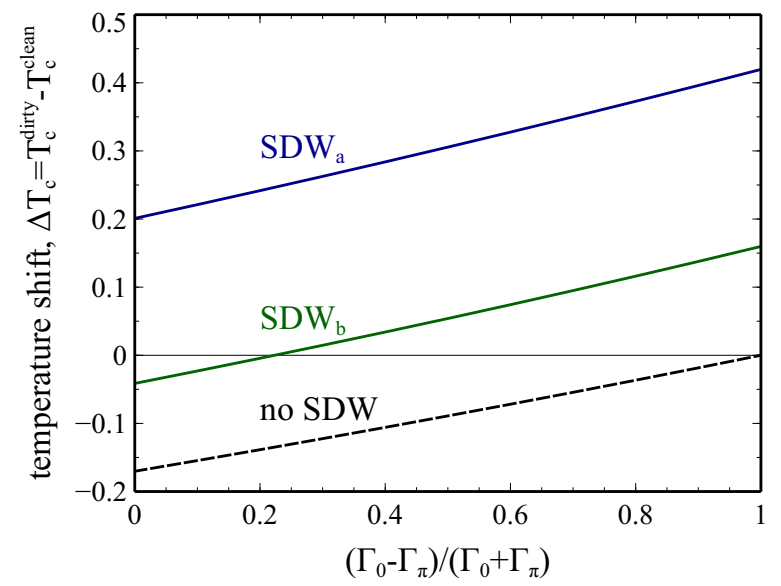

Figure 2: (Color online) $\Delta T_{c}=T_{c}^{\text {dirty }}-T_{c}^{\text {clean }}$ (in units of $\left.T_{c}^{\text {clean }}\right)$ as function of the ratio between intra-band and inter-band impurity scattering amplitudes $\left(\Gamma_{0}\right.$ and $\Gamma_{\pi}$, respectively). Line $\mathrm{SDW}_{\mathrm{a}}$ is for the parameters of Fig. 1(a) at a fixed $\delta_{0} /\left(2 \pi T_{c, 0}\right)=0.2$ and line $\mathrm{SDW}_{\mathrm{b}}$ is for the parameters of Fig. 1(b), at a fixed $\delta_{0} /\left(2 \pi T_{c, 0}\right)=0.6$. For both curves the system is in the coexistence region and $\left(\Gamma_{0}+\Gamma_{\pi}\right) /\left(2 \pi T_{c}^{\text {clean }}\right)=0.01$. The lower curve is for a pure superconductor, $M=0$. In the coexistence region, $\Delta T_{c}$ is definitely positive when $\Gamma_{\pi}$ is small. For $\Gamma_{0} \sim \Gamma_{\pi}$, the behavior of $\Delta T_{c}$ is a result of the competition between the direct pair-breaking effect of impurities, which tends to reduce $T_{c}$, and the suppression of the SDW order parameter, which tends to increase $T_{c}$.

where the operators $\hat{c}(\hat{f})$ refer to electrons near the hole (electron) pocket and the band dispersions are given by $\varepsilon_{1, \mathbf{k}}=-k^{2} /(2 m)+\mu_{h}$ and $\varepsilon_{2, \mathbf{k}}=k_{x}^{2} /\left(2 m_{x}\right)+k_{y}^{2} /\left(2 m_{y}\right)-\mu_{e}$. For small ellipticity $\left|m_{x}-m_{y}\right| \ll m$, the latter can be conveniently parametrized by $\xi_{\mathbf{k}}=\left(\varepsilon_{1, \mathbf{k}}-\varepsilon_{2, \mathbf{k}}\right) / 2 \approx \mathbf{v}_{F}$. $\left(\mathbf{k}-\mathbf{k}_{F}\right)$ and $\delta_{\mathbf{k}}=\left(\varepsilon_{1, \mathbf{k}}+\varepsilon_{2, \mathbf{k}}\right) / 2=\left(\mu_{h}-\mu_{e}\right) / 2+$ $\left(k_{x}^{2}-k_{y}^{2}\right)\left(m_{x}-m_{y}\right) / 4 m \approx \delta_{0}+\delta_{2} \cos \varphi$. We consider the interactions between the low-energy fermions in the SDW (particle-hole) and SC (particle-particle) channels, as well as their interaction with non-magnetic impurities. We first introduce the SDW order parameter $M \propto \sum_{\mathbf{k}, \sigma} \sigma\left\langle c_{\mathbf{k} \sigma}^{\dagger} f_{\mathbf{k} \sigma}\right\rangle$ and reduce the four-fermion SDW interaction to

$$
\mathcal{H}_{\mathrm{SDW}}=M\left(\sum_{\mathbf{k}} \sum_{\sigma= \pm 1} \sigma c_{\mathbf{k} \sigma}^{\dagger} f_{\mathbf{k} \sigma}+\text { h.c. }\right)
$$

where $M$ is obtained self-consistently (see Eq. (5) below). Introducing the Nambu operators $\Psi_{\mathrm{k}}^{\dagger}=$ $\left(c_{\mathbf{k} \uparrow}^{\dagger}, c_{-\mathbf{k} \downarrow}, f_{\mathbf{k} \uparrow}^{\dagger}, f_{-\mathbf{k} \downarrow}\right)$, the bare Green's function is expressed as

$$
\hat{\mathcal{G}}_{0}^{-1}=\left(\begin{array}{cc}
i \omega_{n} \hat{\tau}_{0}-(\xi+\delta) \hat{\tau}_{z} & M \hat{\tau}_{0} \\
M \hat{\tau}_{0} & i \omega_{n} \hat{\tau}_{0}+(\xi-\delta) \hat{\tau}_{z}
\end{array}\right)
$$

where $\hat{\tau}_{i}$ are Pauli matrices in Nambu space. In the Born approximation, impurity scattering gives rise to the 

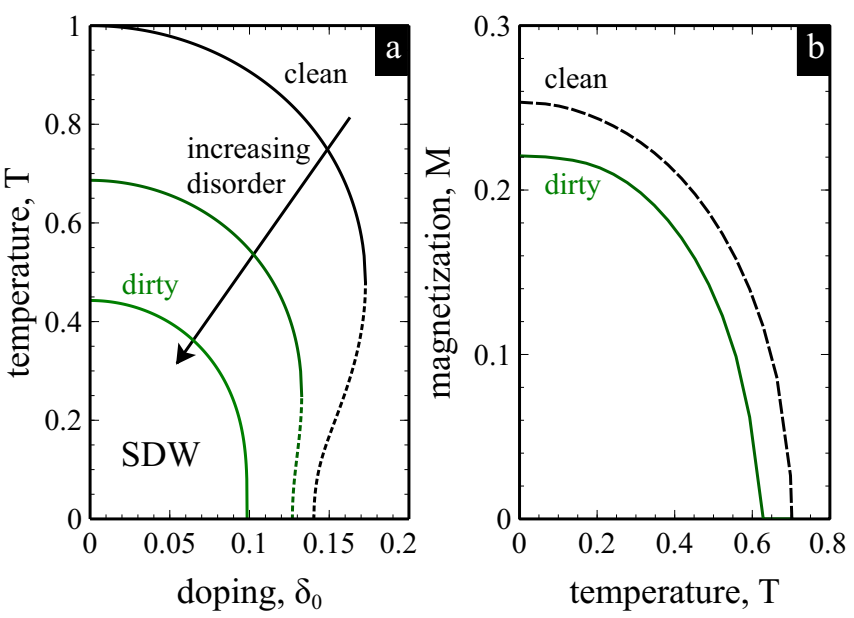

Figure 3: (Color online) (a) SDW transition temperature (in units of $T_{N, 0}$ ) as function of doping $\delta_{0}$ (in units of $2 \pi T_{N, 0}$ ) for the clean $(\gamma=0)$ and dirty cases $\left(\gamma / 2 \pi T_{N, 0}=0.06\right.$ and 0.1$)$. We set $\delta_{2}=0$, but the behavior at a finite $\delta_{2}$ is quite similar. The dashed lines denote metastable solutions of the SDW gap equations, characteristic of the first-order character of the transition. In the dirty case, the second-order SDW transition extends to $T=0$ above a certain threshold of scattering amplitude. (b) Magnetization $M$ (in units of $\left.2 \pi T_{N, 0}\right)$ as function of temperature for $\delta_{0} /\left(2 \pi T_{N, 0}\right)=0.1$ and $\delta_{2} /\left(2 \pi T_{N, 0}\right)=0.2$ (same as in Fig. 1(a)). The dashed and solid lines are for the clean and dirty cases, respectively (in the dirty case $\left.\gamma /\left(2 \pi T_{N, 0}\right)=0.006\right)$.

self-energy correction $\hat{\Sigma}=n_{\text {imp }} \sum_{\mathbf{k}} \hat{U}_{\mathbf{q}-\mathbf{k}} \hat{\mathcal{G}}_{\mathbf{k}} \hat{U}_{\mathbf{k}-\mathbf{q}}$, where $n_{\text {imp }}$ is the density of impurities, $\hat{\mathcal{G}}^{-1}=\hat{\mathcal{G}}_{0}^{-1}-\hat{\Sigma}$ is the renormalized Green's function, and $\hat{U}$ is the impurity potential, which we decompose into an intra-band contribution $u_{0}$ and an inter-band contribution $u_{\pi}$ :

$$
\hat{U}=\left(\begin{array}{ll}
u_{0} \hat{\tau}_{z} & u_{\pi} \hat{\tau}_{z} \\
u_{\pi} \hat{\tau}_{z} & u_{0} \hat{\tau}_{z}
\end{array}\right)
$$

To find $\hat{\mathcal{G}}$, we write it in the same form as Eq. (3), but with renormalized parameters $\tilde{\omega}_{n}, \tilde{\delta}_{0}$, and complex $\tilde{M}$, with $\mathcal{G}_{13}^{-1}=\mathcal{G}_{31}^{-1}=\tilde{M}$ and $\mathcal{G}_{24}^{-1}=\mathcal{G}_{42}^{-1}=\tilde{M}^{*}$. The parameter $\delta_{2}$ retains its bare value since $\left\langle\delta_{2} \cos 2 \varphi\right\rangle=$ 0 across the Fermi surface. Introducing the scattering amplitudes $\Gamma_{i}=\pi N_{F} n_{\mathrm{imp}} u_{i}^{2}$, we obtain a set of selfconsistent equations

$$
\begin{aligned}
\tilde{\nu}_{n} & =\nu_{n}+\gamma\left(\tilde{\nu}_{n} \tilde{\Pi}_{1}+i \tilde{\Pi}_{2}\right) \\
\tilde{M} & =M-\tilde{M} \gamma \tilde{\Pi}_{1} \\
M & =\lambda_{\text {sdw }} T \sum_{n} \operatorname{Re}\left(\tilde{M} \tilde{\Pi}_{1}\right)
\end{aligned}
$$

where $\nu_{n}=\omega_{n}+i \delta_{0}, \gamma=\Gamma_{0}+\Gamma_{\pi}, M$ is the (real) SDW order parameter affected by impurities, and $\lambda_{\mathrm{sdw}}$ is the SDW coupling constant. The Neel transition temperature $T_{N, 0}$ to a pure SDW phase at perfect nesting and zero disorder is related to $\lambda_{\mathrm{sdw}}$ by $1 / \lambda_{\mathrm{sdw}}=\ln \left(\Lambda / T_{N, 0}\right)$, where $\Lambda$ is the high-energy cutoff. We emphasize that both intra-band and inter-band impurity scattering affect $T_{N}$ and the order parameter in the SDW channel. We also introduce

$$
\tilde{\Pi}_{1} \equiv \int \frac{d \varphi}{2 \pi} \frac{1}{\tilde{\Omega}}, \quad \tilde{\Pi}_{2} \equiv \int \frac{d \varphi}{2 \pi} \frac{\cos 2 \varphi}{\tilde{\Omega}}
$$

with $\tilde{\Omega}=\sqrt{\tilde{M}^{2}+\left(\tilde{\nu}_{n}+i \delta_{2} \cos 2 \varphi\right)^{2}}$. Note that $\tilde{\Pi}_{i}$ by itself depends on the renormalized variables, i.e. Eqs. (5) are non-linear self-consistent equations.

The solution of the set (5) gives the Green's function of a dirty SDW magnet, which then acts as a bare Green's function for the SC system. Since we are only interested in $T_{c}$, we restrict our analysis to the linearized $\mathrm{SC}$ gap equation in the presence of impurities and a non-zero SDW order parameter:

$$
\frac{1}{T_{c}}=\lambda_{\mathrm{sc}} \sum_{n} \frac{\tilde{\Pi}_{3}}{1-\zeta \tilde{\Pi}_{3}}
$$

where $\zeta=\Gamma_{0}-\Gamma_{\pi}, 1 / \lambda_{\mathrm{sc}}=\ln \left(\Lambda / T_{c, 0}\right)$ is the coupling constant in the $\mathrm{SC}$ channel, $T_{c, 0}$ is the superconducting transition temperature without SDW and at perfect nesting, and

$\tilde{\Pi}_{3}=\int \frac{d \varphi}{2 \pi}\left(\frac{1}{\tilde{\Omega}+\tilde{\Omega}^{*}}\right)\left(1+\frac{\left|\tilde{\nu}_{n}+i \delta_{2} \cos 2 \varphi\right|^{2}+|\tilde{M}|^{2}}{|\tilde{\Omega}|^{2}}\right)$

Equation (7) reduces to the gap equation of an ordinary dirty $s^{+-}$superconductor if we set $M=\tilde{M}=0$. Alternatively, at perfect nesting, $\delta_{0}=\delta_{2}=0$, we recover the results of Ref 18 .

Results and comparison to experiments. We first consider the pure SDW state. In Fig. 3 we show that both the SDW transition temperature $T_{N}$ and the order parameter $M$ are reduced in the presence of impurities. This behavior is entirely expected, since both intraband and inter-band impurity scattering are detrimental to SDW. A less obvious result is that impurities also affect the character of the SDW transition at low $T$. In the clean case, the SDW transition is first-order at low enough $T \stackrel{14}{\underline{14}}$ Impurities add additional scattering and effectively shift $T \rightarrow T+\gamma$, extending the range of the second-order transition to smaller temperatures $T$. Once $\gamma$ exceeds a critical value $\gamma_{c r}$, the second-order transition line extends down to $T=0$. The critical $\gamma_{c r}$ is obtained in a straightforward way by expanding the last equation in (5) to order $M^{3}$ and verifying when the cubic coefficient changes sign. For $\delta_{2}=0$, we obtain analytically $\gamma_{c r} /\left(2 \pi T_{N, 0}\right) \approx 0.08$, where $T_{N, 0}$ is the SDW transition temperature at perfect nesting.

We now use the SDW results as input and solve Eq. (7) for $T_{c}$. To verify whether $T_{c}$ is reduced or enhanced with increasing disorder, it is sufficient to consider small $\Gamma_{0}$ and $\Gamma_{\pi}$ and evaluate $\Delta T_{c}=T_{c}^{\text {dirty }}-T_{c}^{\text {clean }}$ to first order in 
$\Gamma_{i} / T_{N, 0}$. The computations are tedious but straightforward, so we skip the details and present our results. The phase diagram in the presence of impurity scattering is shown in Fig. 1 for on-site impurity potential $\left(\Gamma_{0}=\Gamma_{\pi}\right)$ and two ratios of $T_{N, 0} / T_{c, 0}$. We clearly see two different types of behavior in the coexistence phase: $T_{c}$ either increases when impurities are added, or decreases at a slow rate. This non-universal behavior can be understood qualitatively: for a small $T_{N, 0} / T_{c, 0}$ (Fig. 1(a)) the effects of disorder on $T_{N}$ and $T_{c}$ are comparable, and the feedback on $T_{c}$ from the reduction of $T_{N}$ overshadows the direct pair-breaking effect on $T_{c}$. For a larger ratio $T_{N, 0} / T_{c, 0}$, the effect of disorder on SDW gets relatively weaker, and the direct pair-breaking effect on $T_{c}$ prevails (more specifically, we estimate that $T_{c}$ increases with disorder when the ratio $T_{N, 0} /\left(\Gamma_{0}+\Gamma_{\pi}\right)$ is smaller than the ratio $\left.T_{c, 0} / \Gamma_{\pi}\right)$.

In Fig. 2 we plot $\Delta T_{c}=T_{c}^{\text {dirty }}-T_{c}^{\text {clean }}$ as a function of the ratio between intra-band and inter-band impurity scattering amplitudes. We consider the two sets of parameters of Fig. 1 with $\delta_{0}$ in the coexistence region and compare them with the case when no SDW is present. In the latter, $\Delta T_{c}<0$ when $\Gamma_{\pi}$ is non-zero. We see that in the coexistence region $\Delta T_{c}$ is definitely positive when $\Gamma_{0} / \Gamma_{\pi}$ is large enough, i.e. $T_{c}$ increases when impurities are added into the coexistence state. As expected, this increase is the largest when $\Gamma_{\pi}$ vanishes, since in this limit impurities are not pair-breaking, but still suppress $M$. When $\Gamma_{0}$ and $\Gamma_{\pi}$ are comparable, $T_{c}$ can either increase or decrease, depending on parameters, but even when it decreases, the rate of the decrease is smaller than that for a pure SC state.

Our results offer an explanation for the non-monotonic behavior of $\Delta T_{c}=T_{c}^{\text {dirty }}-T_{c}^{\text {clean }}$ as function of doping observed in Refs. [9, 11] by adding $\mathrm{Zn}$ to $\mathrm{LaFeAs}\left(\mathrm{O}_{1-x} \mathrm{~F}_{x}\right)$ and $\mathrm{Cu}$ to $\mathrm{Ba}\left(\mathrm{Fe}_{1-x} \mathrm{Co}_{x}\right)_{2} \mathrm{As}_{2}$, respectively. They also offer an explanation for the observation in Ref. [13] that the addition of $\mathrm{Zn}$ to underdoped $\mathrm{Ba}\left(\mathrm{Fe}_{1-x} \mathrm{Co}_{x}\right)_{2} \mathrm{As}_{2}$ leads to a decrease of $\Delta T_{c}$, but at a slower rate than in the overdoped region. For this material, the fact that $\mathrm{Zn}$ substitution leads to $\Delta T_{c}<0$ while $\mathrm{Cu}$ substitution leads to $\Delta T_{c}>0$ may be due to different disorder potentials associated with each dopant, leading to different ratios $\Gamma_{0} / \Gamma_{\pi}$. It is also possible that the foreign element not only acts as an impurity but also changes the electronic chemical potential and/or the Fermi surface geometry.

We caution that the microscopic coexistence of SC and SDW orders has been well-established for 122 compounds like $\mathrm{Ba}\left(\mathrm{Fe}_{1-x} \mathrm{Co}_{x}\right)_{2} \mathrm{As}_{2}$, but this issue has not been settled for the 1111 systems like $\mathrm{LaFeAs}\left(\mathrm{O}_{1-x} \mathrm{~F}_{x}\right)$. Yet, even if SDW and SC phase-separate and occupy different parts of the sample, we expect some of the physics described here to hold, i.e. that impurity scattering on the one hand is pair-breaking and on the other hand tends to increase $T_{c}$ by suppressing the competing SDW phase.

Conclusions. In summary, we showed that the different behaviors of $T_{c}$ with impurity scattering observed in overdoped and underdoped iron-based superconductors can be understood within the $s^{+-}$scenario for superconductivity. While in the overdoped regime $T_{c}$ is quickly reduced with increasing impurity scattering, in the underdoped regime there are two competing effects: the direct pair-breaking by impurities, which reduces $T_{c}$, and the suppression of the coexisting SDW order parameter, which increases $T_{c}$. We demonstrated that, due to competition between these two effects, $T_{c}$ in the coexistence region either drops at a smaller rate or even increases with increasing impurity concentration, in agreement with the experimental data. We view this agreement as an evidence that the gap symmetry in the iron pnictides is indeed $s^{+-}$.

We thank E. Bascones, S. Bud'ko, P. Canfield, F. Hardy, I. Eremin, A. Kaminski, S. Maiti, Y. Matsuda, N. Ni, R. Prozorov, J. Schmalian, M. Tanatar, A. Vorontsov, and Zhu-an $\mathrm{Xu}$ for useful discussions. R.M.F. acknowledges the support from ICAM and nsf-dmr 0645461, as well as the valuable support from the NSF Partnerships for International Research and Education (PIRE) program OISE-0968226. M.G.V. and A.V.C. are supported by nsf-dmr 0955500 and 0906953, respectively. R.M.F. and A.V.C. thank the hospitality of the Aspen Center for Physics, where part of this work has been done.
1 D. C. Johnston, Adv. Phys. 59, 803 (2010); J. Paglione and R. L. Greene, Nature Phys. 6, 645 (2010). P. J. Hirschfeld, M. M. Korshunov, and I. I. Mazin, Rep. Prog. Phys. 74, 124508 (2011); D. N. Basov and A. V. Chubukov, Nature Phys. 7, 241 (2011); P. C. Canfield and S. L. Bud'ko, Annu. Rev. Cond. Mat. Phys. 1, 27 (2010); H. H. Wen and S. Li, Annu. Rev. Cond. Mat. Phys. 2, 121 (2011); A. V. Chubukov, Annu. Rev. Cond. Mat. Phys. 3, 57 (2012); G. R. Stewart, Rev. Mod. Phys. 831589 (2011).

2 H. Ding, P. Richard, K. Nakayama, K. Sugawara, T. Arakane, Y. Sekiba, A. Takayama, S. Souma, T. Sato, T. Takahashi, Z. Wang, X. Dai, Z. Fang, G. F. Chen, J. L. Luo and N. L. Wang, Euro. Phys. Lett. 8347001 (2008); T. Kondo, A. F. Santander-Syro, O. Copie, C. Liu, M. E. Tillman, E. D. Mun, J. Schmalian, S. L. Bud'ko, M. A.
Tanatar, P. C. Canfield, and A. Kaminski, Phys. Rev. Lett. 101, 147003 (2008); S. V. Borisenko, V. B. Zabolotnyy, D. V. Evtushinsky, T. K. Kim, I. V. Morozov, A. N. Yaresko, A. A. Kordyuk, G. Behr, A. Vasiliev, R. Follath, and B. Buchner, Phys. Rev. Lett. 105, 067002 (2010); T. Shimojima, F. Sakaguchi, K. Ishizaka, Y. Ishida, T. Kiss, M. Okawa, T. Togashi, C.-T. Chen, S. Watanabe, M. Arita, K. Shimada, H. Namatame, M. Taniguchi, K. Ohgushi, S. Kasahara, T. Terashima, T. Shibauchi, Y. Matsuda, A. Chainani, and S. Shin, Science 332, 564 (2011).

3 S. Maiti, M. M. Korshunov, T. A. Maier, P. J. Hirschfeld, and A. V. Chubukov, Phys. Rev. B 84, 224505 (2011); Phys. Rev. Lett. 107, 147002 (2011).

4 I. I. Mazin, D. J. Singh, M. D. Johannes, and M. H. Du, Phys. Rev. Lett. 101, 057003 (2008); K. Kuroki, S. Onari, 
R. Arita, H. Usui, Y. Tanaka, H. Kontani, and H. Aoki, Phys. Rev. Lett. 101, 087004 (2008); J. Zhang, R. Sknepnek, R. M. Fernandes, and J. Schmalian, Phys. Rev. B 79, 220502(R) (2009); A.F. Kemper, T.A. Maier, S. Graser, H-P. Cheng, P.J. Hirschfeld and D.J. Scalapino, New J. Phys. 12, 073030 (2010).

5 S. Onari and H. Kontani, Phys. Rev. Lett. 103, 177001 (2009); H. Kontani and S. Onari, Phys. Rev. Lett. 104, 157001 (2010); Y. Yanagi, Y. Yamakawa, and Y. Ono, Phys. Rev. B 81, 054518 (2010); T. Saito, S. Onari, and H. Kontani Phys. Rev. B 83, 140512(R) (2011).

6 A. V. Chubukov, D. V. Efremov and I Eremin, Phys. Rev. B 78, 134512 (2008); O. V. Dolgov, A. A. Golubov, D. Parker, New Journal of Physics, 11, 075012 (2009); A. B. Vorontsov, M. G. Vavilov, and A. V. Chubukov, Phys. Rev. B 79, 140507 (2009); Y. Bang, Europhys. Letters, 86, 47001 (2009); V.G. Kogan, Phys. Rev. B 80, 214532 (2009); K. Nakamura, R. Arita, and H. Ikeda, Phys. Rev. B 83, 144512 (2011).

7 R. S. Dhaka, C. Liu, R. M. Fernandes, R. Jiang, C. P. Strehlow, T. Kondo, A. Thaler, J. Schmalian, S. L. Bud'ko, P. C. Canfield, and A. Kaminski, Phys. Rev. Lett. 107, 267002 (2011); K. Kirshenbaum, S.R. Saha, S. Ziemak, T. Drye, and J. Paglione, arXiv:1203.5114

8 Y. K. Li, X. Lin, Q. Tao, C. Wang, T. Zhou, L. Li, Q. Wang, M. He, G. Cao, and Z. A. Xu, New J. Phys. 11, 053008 (2009).

${ }^{9}$ Y. K. Li, J. Tong, Q. Tao, C. Feng, G. Cao, W. Chen, F. C. Zhang, and Z. A. Xu, New J. Phys. 12, 083008 (2010).

10 Y. F. Guo, Y. G. Shi, S. Yu, A. A. Belik, Y. Matsushita, M. Tanaka, Y. Katsuya, K. Kobayashi, I. Nowik, I. Felner, V.
P. S. Awana, K. Yamaura, and E. Takayama-Muromachi, Phys. Rev. B 82, 054506 (2010).

11 N. Ni, A. Thaler, J. Q. Yan, A. Kracher, E. Colombier, S. L. Bud'ko, and P. C. Canfield, Phys. Rev. B 82, 024519 (2010).

12 M. G. Kim et al, arXiv:1204.1538

13 J. Li, Y. Guo, S. Zhang, Y. Tsujimoto, X. Wang, C. I. Sathish, S. Yu, K. Yamaura, and E. Takayama-Muromachi, Solid State Commun. 152, 671 (2012).

14 A. B. Vorontsov, M. G. Vavilov, and A. V. Chubukov, Phys. Rev. B 81, 174538 (2010), Phys. Rev. B 84, 140502 (2011).

15 R. M. Fernandes, D. K. Pratt, W. Tian, J. Zarestky, A. Kreyssig, S. Nandi, M. G. Kim, A. Thaler, N. Ni, P. C. Canfield, R. J. McQueeney, J. Schmalian, and A. I. Goldman, Phys. Rev. B 81, 140501 (2010); R. M. Fernandes and J. Schmalian, Phys. Rev. B 82, 014520 (2010); ibid Phys. Rev. B 82, 014521 (2010).

16 M. T. Rice, Phys. Rev. B 2, 3619 (1970); N. Kulikov and V.V.Tugushev, Sov. Phys. Usp. 27, 954 (1984), [Usp.Fiz.Nauk 144643 (1984)]; V. Cvetkovic and Z. Tesanovic, Europhys. Lett. 85, 37002 (2009).

17 Two of us recently used the fact that both SDW and SC are affected by impurities to argue that the phase diagram in case when doping acts as a non-magnetic impuritry is the same as when doping modifies the band structure ${ }^{18}$

18 M.G. Vavilov and A. V. Chubukov, Phys. Rev. B 84, 214521 (2011).

19 I. Eremin and A.V. Chubukov, Phys. Rev. B 81, 024511 (2010). 